\title{
Kaempferol pretreatment modulates systemic inflammation and oxidative stress following hemorrhagic shock in mice
}

\author{
Qi-Sheng Yang ${ }^{1}$, Li-Ping He${ }^{2}$, Xian-Long Zhou' ${ }^{1}$, Yan Zhao ${ }^{1 *}$, Jun Shen ${ }^{1}$, Peng Xu ${ }^{1}$ and Shao-Zhou $\mathrm{Ni}^{1}$
}

\begin{abstract}
Background: Kaempferol has been reported as beneficial for both acute and chronic inflammatory diseases. This study aims to investigate whether kaempferol affects systemic inflammation and oxidative stress in the heart, lung, and liver after hemorrhagic shock in mice.

Methods: Male C57/BL6 mice underwent hemorrhagic shock (mean arterial pressure of $35 \mathrm{mmHg}$ for $90 \mathrm{~min}$ ) and were arbitrarily divided into Sham, hemorrhagic shock (HS), and Kae groups ( $n=10$ in each group). Mice in the Kae groups received a kaempferol (10-mg/kg body weight) injection $12 \mathrm{~h}$ prior to (Group Kae PT) or 90 min after (Group Kae T) the initiation of hemorrhagic shock. Plasma proinflammatory cytokines (TNF-a and IL-6), organ myeloperoxidase (MPO) and superoxide dismutase (SOD) activities, and organ malondialdehyde (MDA) concentrations and heme oxygenase-1 (HO-1) expression levels were assessed by enzyme-linked immunosorbent assay (ELISA) or western blot assay.

Results: Compared with the HS group and the Kae T group, pretreatment with kaempferol significantly decreased proinflammatory cytokines TNF-a $(P=0.012$ and 0.015 , respectively) and IL-6 $(P=0.023$ and 0.014 , respectively) following hemorrhagic shock. Kae pretreatment reverted MPO, SOD, and MDA to basal levels in the heart, lung, and liver $\left(P_{S}<0.05\right)$, while the Kae T group showed no significant differences in these biomarkers compared with the HS group (PS > 0.05). HO-1 expression was significantly increased in the Kae PT group compared with the other groups ( $P=0.011$ vs. HS group and $P=0.02$ vs. Kae T group).

Conclusions: Pretreatment of hemorrhagic shock mice with kaempferol significantly decreased plasma levels of TNF- $a$ and IL-6; reverted MPO, SOD, and MDA in the heart, lung, and liver; and increased expression of HO-1 in the same organs.
\end{abstract}

Keywords: Hemorrhagic shock, Kaempferol, Oxidative stress, Systematic inflammation

\section{Background}

Hemorrhagic shock (HS) is one of the most common causes of deaths related to severe trauma [1,2]. HS is generally characterized by hemodynamic instability with cellular hypoxia and diminishing organ function [3]. Trauma and HS may stimulate a systemic release of endogenous molecules and provoke local and systemic liberation of both pro- and anti-inflammatory cytokines such as interleukin-1 beta (IL-1 $\beta)$, IL-2, IL-4, and tumor

\footnotetext{
*Correspondence: doctoryanzhao@163.com

${ }^{1}$ Emergency Center, Zhongnan Hospital of Wuhan University, Wuhan, Hubei 430071, China

Full list of author information is available at the end of the article
}

necrosis factor-alpha (TNF- $\alpha)[4,5]$. Thus, severe and developing HS ultimately results in systematic inflammatory response syndrome or multiple organ failure and even death [6]. In addition, oxidative stress is implicated in the high mortality and morbidity of HS [6], and treatment with antioxidative stress agents could improve the outcome of HS [7-9].

Kaempferol is believed to have anti-inflammatory activities. It has been shown to have potential immunemodulatory effects and to interfere with a number of inflammation mechanisms in vitro [10,11]. Kowalski et al. [11] demonstrated that kaempferol blocks expression of both IL-1 $\beta$ and TNF- $\alpha$ in macrophages. Kaempferol 
significantly prohibits TNF assembly [12], and inhibits IL-2- and IL-4-mediated inflammatory outcomes [12,13]. Although both antioxidant and pro-oxidant activities of flavonoids have been previously studied $[14,15]$, the antioxidant or pro-oxidant ability of kaempferol in HS has not been investigated.

This study aims to investigate whether kaempferol affects systematic inflammation and oxidative stress in the heart, lung, and liver after HS in mice.

\section{Methods and materials}

\section{Animals}

Forty 8-10-week-old specific pathogen-free male C57/ BL6 mice weighing 20-25 g were purchased from the Center for Animal Experiment of Wuhan University (Wuhan, Hubei, China). The Research Committee of Wuhan University approved this study (No. 20130037). All experiments were conducted in accordance with the guidelines of the Animal Use and Care Committee of Wuhan University. Mice were housed individually in cages on a 12 -h day/12-h night cycle at $23-27^{\circ} \mathrm{C}$ and had free access to food and water.

\section{Animal model}

An HS model was used as previously described [16]. Briefly, animals were anesthetized with an intraperitoneal (i.p.) injection of pentobarbital sodium $(50-\mathrm{mg} / \mathrm{kg}$ body weight [BW]; Amresco, Cleveland, OH, USA). Cannulation was performed using sterile polyethylene tubing on both right and left femoral arteries. All the catheters and syringes were pretreated with heparinized normal saline (25 IU/mL, Shuanghe, Beijing, China). The right femoral artery cannulation tube was connected with a pressure transducer to the $\mathrm{BL}-420 \mathrm{~F}$ biological signal acquisition system (Chengdu Taineng Ltd., Chengdu, China). Mean arterial pressure (MAP) was recorded throughout this experiment. HS was performed by withdrawing blood over a 15-min interval via the left femoral artery cannulation tube. MAP was controlled at $30-40 \mathrm{mmHg}$ for 90 min followed by 15-min fluid resuscitation using an equal volume of normal saline as lost blood. Thereafter, the catheter was removed, the artery ligated, and the skin incision closed. After a recovery phase of $4.5 \mathrm{~h}$, the mice were sacrificed. Blood samples, and heart, lung, and liver tissue were collected for biochemical analysis.

\section{Group distribution}

Mice were arbitrarily divided into four groups: (1) a Sham group $(\mathrm{n}=10)$ : animals received femoral artery catheterizations without drawing blood; (2) a hemorrhagic shock (HS) group $(n=10)$ : animals underwent HS and received an i.p. injection of $100-\mu \mathrm{L}$ phosphate-buffered saline (PBS) (Boster, Wuhan, China) at the end of the 90-min shock period; (3) a kaempferol pretreatment (Kae PT) group $(\mathrm{n}=10)$ : animals received an injection of kaempferol (Sigma, St. Louis, MO, USA) (i.p., $10 \mathrm{mg} / \mathrm{kg}$ BW, dissolved in $100 \mu \mathrm{L}$ of PBS) $12 \mathrm{~h}$ prior to the initiation of HS; and (4) a kaempferol treatment (Kae T) group $(n=10)$ : HS animals received a kaempferol injection (i.p., $10 \mathrm{mg} / \mathrm{kg}$ BW in $100 \mu \mathrm{L}$ of PBS) at the end of the shock period (Figure 1).

\section{Plasma TNF- $\alpha$ and IL- 6 measurements}

At the end of the study, blood samples were collected by cardiac puncture. Heparinized blood samples were centrifuged (5415D, Eppendorf, Germany) at $1200 \times g$ for $10 \mathrm{~min}$ at $4^{\circ} \mathrm{C}$. Thereafter, supernatants were stored at $-80^{\circ} \mathrm{C}$ until measurements. Plasma cytokines including TNF- $\alpha$ (Cat no. MTA00B) and IL-6 (Cat no. M6000B) were measured by commercial enzyme-linked immunosorbent assay (ELISA) kits (R\&D Systems Inc., Minneapolis, MN, USA) according to the manufacturer's protocols.

\section{Myeloperoxidase activity}

Fresh heart, lung, and liver tissues were frozen at the end of the study. Tissue was homogenized in a lysis buffer before the determination of myeloperoxidase (MPO) activity. MPO activity was determined by a commercial ELISA kit (Cat no. LS-F278-1, Biocompare, CA, USA) according to the manufacturer's protocols. Previous studies $[16,17]$ have reported observations of only minor histological changes following HS models. Therefore, we did not perform histological evaluation in this study.

\section{Malondialdehyde levels}

Fresh tissue was placed into a $1.5-\mathrm{ml}$ centrifuge tube. RIPA Buffer $250 \mu \mathrm{L}$ was added with protease inhibitors. Homogenate was then centrifuged at $11000 \times g$ for $10 \mathrm{~min}$ at $4^{\circ} \mathrm{C}$. The supernatant was used for determining malondialdehyde (MDA) by a commercial kit (Cat no. 10009055, Cayman, Ann Arbor, MI).

\section{Superoxide dismutase activity}

The supernatant of homogenate after centrifuge $(12,000 \times$ g, $10 \mathrm{~min}$; 5415D, Eppendorf, Germany) was used for detecting superoxide dismutase (SOD) activity. Tissue SOD activity was measured with a SOD-525TM reagent kit (OXIS International, Foster, CA, USA); the final result was expressed as $\mathrm{U} / \mathrm{mg}$ protein.

\section{Heme oxygenase- 1 expression in organs}

Western blot assay was used to detect the expression of heme oxygenase-1 (HO-1) in the main organs after HS. Briefly, 40- $\mu$ g protein extracts were loaded onto a $10 \%$ resolving gel for electrophoresis. Proteins were transblotted onto membranes (Millipore, Temecula, CA, USA). Then, membranes were blocked for $1 \mathrm{~h}$ at room temperature. The blot was immune-probed overnight 


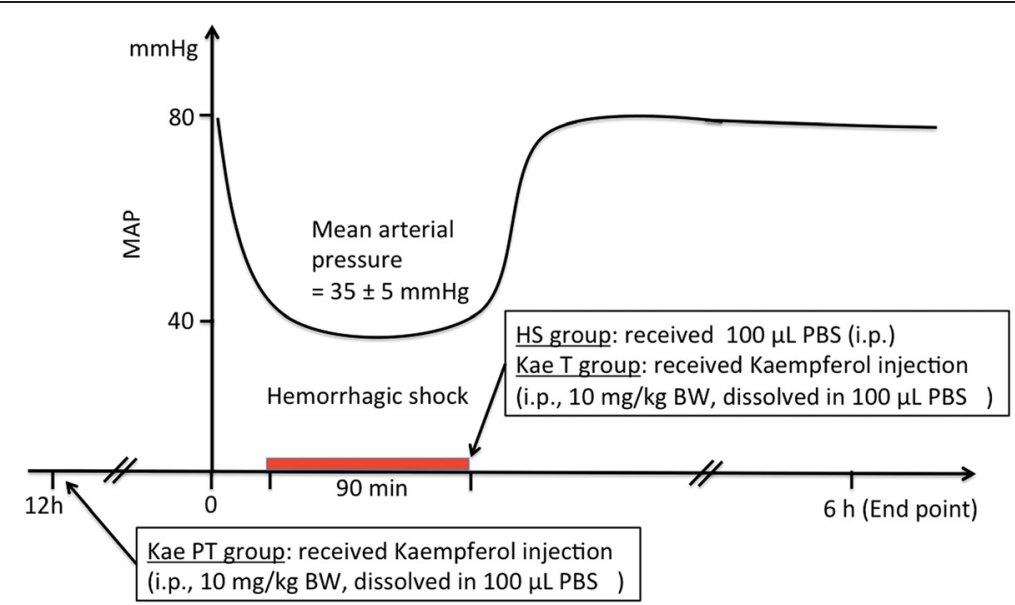

Figure 1 Study protocol. Animals were assigned to Sham $(n=10), H S(n=10)$, Kae T $(n=10)$, and Kae PT $(n=10)$ groups. The HS group received an injection of 100- $\mu$ L PBS (i.p.) at the end of the shock period. Kae T and Kae PT groups received an injection of kaempferol (i.p., 10 mg/kg BW, dissolved in 100- $\mu \mathrm{L}$ PBS) 90 min following or $12 \mathrm{~h}$ prior to the initiation of HS, respectively. Animals were sacrificed $6 \mathrm{~h}$ after the initiation of HS for blood and tissue sample collection.

with primary antibodies including HO-1 (1:2000; Abcam, Cambridge, MA, USA) and $\beta$-actin (1:1000; Santa Cruz Biotechnology, Dallas, TX, USA). The blots were incubated with a secondary antibody for $1 \mathrm{~h}$ at room temperature. The signals were detected by enhanced chemiluminescene (ECL) and quantified by Photoshop CS6 software (Adobe, USA).

\section{Statistical analysis}

Data were processed using the statistical analysis software SPSS version 18.0 (SPSS Inc., Chicago, IL, USA) and were expressed as mean $\pm \mathrm{SD}$. Comparisons of several means were performed using one-way and repeated measures two-way analysis of variance followed by the Tukey-Kramer test to identify significant differences between groups. All statistical significance tests were two-tailed and $P$ values of less than 0.05 were considered significant.

\section{Results}

\section{General characteristics of mice}

The general characteristics of animals were shown in Table 1. The average BW and age were similar for all groups. MAP was recorded throughout the experiments.
MAP during the HS period (MAP-in-HS) and after fluid resuscitation (MAP-post-HS) was calculated. MAP dramatically decreased after blood withdrawals, and MAP in the HS period was well controlled at 30-40 $\mathrm{mmHg}$. There were no significant differences in MAP-in-HS and MAP-post-HS between the HS, Kae PT, and Kae T groups (HS vs. Kae PT, $P=0.14$; Kae PT vs. Kae T, $P=0.32$; HS vs. Kae T, $P=0.10$ ). However, both MAP-in-HS and MAP-post-HS were significantly higher in the Sham group than in the HS, Kae PT, and Kae T groups (MAP-in-HS: $P=0.001,0.002$, and 0.001, respectively and MAP-post-HS: $P=0.012,0.011$, and 0.036 , respectively).

\section{Measurements of plasma cytokines}

Compared with the Sham group, the plasma levels of TNF- $\alpha$ and IL- 6 were significantly increased after HS in the HS $(P=0.001)$, Kae PT $(P=0.001)$, and Kae T $(P=0.0034)$ groups (Figure 2$)$. The i.p. injection of kaempferol $12 \mathrm{~h}$ prior to the initiation of HS inhibited the release of both TNF- $\alpha(P=0.012 v s$. HS; $P=0.015$ vs. Kae T) and IL-6 ( $P=0.023$ vs. HS; $P=0.014$ vs. Kae T), while the injection of kaempferol 90 min following HS affected neither the plasma TNF- $\alpha$ nor the IL- 6 levels.

Table 1 General characteristics in all groups (mean \pm SD)

\begin{tabular}{lllll}
\hline Groups (n) & Age (d) & Body weight $(\mathbf{g})$ & MAP-in-HS $\mathbf{~}^{\mathbf{( m m H g}}$ & MAP-post-HS $^{\mathbf{2}}$ (mmHg) \\
\hline Sham (10) & $63 \pm 5$ & $23.0 \pm 1.5$ & $85.4 \pm 7.5^{\# \#}$ & $82.6 \pm 6.4^{\#}$ \\
HS (10) & $63 \pm 5$ & $22.0 \pm 1.2$ & $36.4 \pm 3.0$ & $74.5 \pm 5.5$ \\
Kae PT (10) & $64 \pm 4$ & $23.3 \pm 1.5$ & $35.0 \pm 2.9$ & $78.8 \pm 6.0$ \\
Kae T (10) & $64 \pm 5$ & $22.1 \pm 2.4$ & $36.2 \pm 3.5$ & $76.5 \pm 7.0$ \\
\hline
\end{tabular}

${ }^{1}$ MAP-in-HS: MAP during a 90-min HS period; ${ }^{2}$ MAP-post-HS: MAP after normal saline resuscitation; MAP was automatically recorded and calculated via a BL-420 $\mathrm{F}$ biological signal acquisition system. No significant differences were found in age and body weight between groups. However, both MAP-in-HS and MAP-post-HS were significantly higher in the Sham group than in the HS, Kae PT, and Kae T groups. Compared with the other groups, ${ }^{\#} P<0.05,{ }^{\#} P<0.01$. 

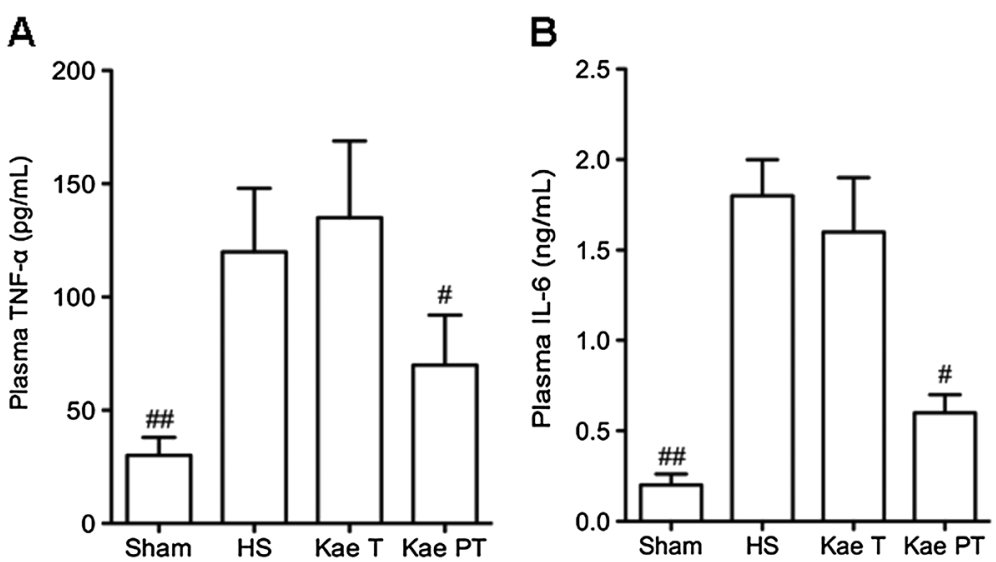

Figure 2 Changes of plasma TNF- $a$ (A) and IL-6 (B) in all groups. Both TNF- $a$ and IL-6 significantly increased after HS. Pretreatment with kaempferol reverted these two cytokines to basal levels. Compared with the other groups, ${ }^{\#} P<0.05,{ }^{\# \#} P<0.01$.

\section{Organ inflammation}

The MPO activities were measured in the heart, lung, and liver (Figure 3). Elevated cardiac, pulmonary, and hepatic MPO activities were observed after $\mathrm{HS}$ in the HS, Kae PT, and Kae T groups. There was no significant difference in MPO activity between the HS and Kae T groups $(P=0.34,0.19$, and 0.074 in the heart, lung, and liver, respectively). However, the MPO activity was significantly decreased in the Kae PT group compared with the HS and Kae T groups (heart: $P=0.018$ and 0.01 ; lung: $P=0.024$ and 0.017 ; liver: $P=0.011$ and 0.031 , respectively).

\section{Organ oxidative response}

We evaluated the MDA levels and SOD activities in the heart, lung, and liver to investigate the effects of kaempferol on oxidative response following HS. As seen in Figure 4, MDA levels were significantly higher in the HS groups than in the Sham group $(P=0.001,0.016$, and 0.011 in the heart, lung, and liver, respectively), while SOD activities were decreased in the HS groups compared with the Sham group $(P=0.001,0.023$, and 0.010 in the heart, lung, and liver, respectively). The injection of kaempferol following HS (Kae T) showed no effects on MDA levels in the heart $(P=0.34)$, lung $(P=0.43)$, and liver $(P=0.11)$ and on SOD activities in the heart $(P=0.16)$, lung $(P=0.37)$, and liver $(P=0.25)$ compared with the HS group. However, the injection of kaempferol $12 \mathrm{~h}$ prior to the induction of HS resulted in decreased MDA levels $(P=0.012,0.001$, and 0.002 , respectively) and increased SOD activities $(P=0.032,0.024$, and 0.004 , respectively) compared with the HS group in the heart, lung, and liver.

\section{Expression of HO-1 in the heart, lung, and liver}

This study found evidence of HO-1 expression in the main organs including the heart, lung, and liver. As seen in Figure 5, HO-1 expression was insignificantly increased in the heart, lung, and liver in the HS group compared with the Sham group $(P=0.37,0.22$, and 0.19 , respectively). The injection of kaempferol following HS showed no effects on HO-1 expression in organs including the heart $(P=0.063)$, lung $(P=0.21)$, and liver $(P=0.37)$ after HS, while the injection of kaempferol $12 \mathrm{~h}$ prior to the induction of HS resulted in significantly increased expression of $\mathrm{HO}-1$ in the heart $(P<0.001)$, lung $(P=0.001)$, and liver $(P<0.001)$ compared with the HS group.
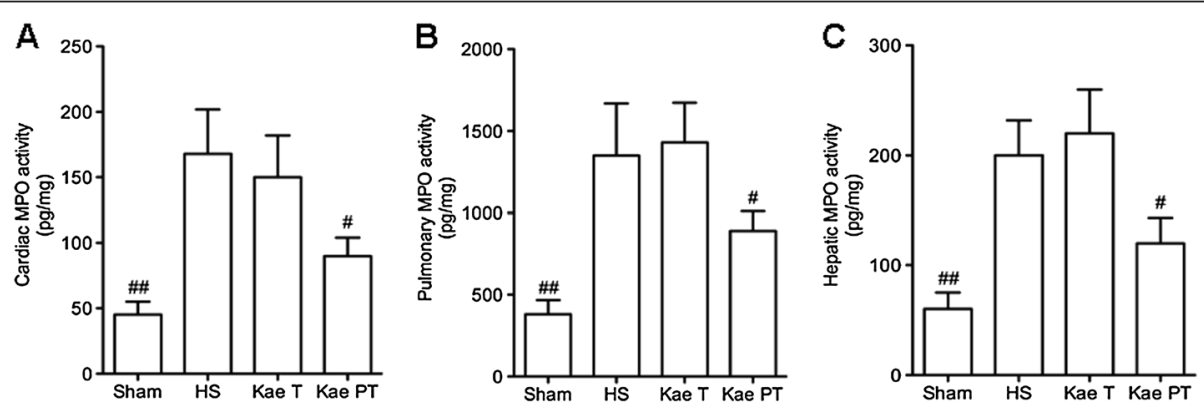

Figure 3 MPO activities in heart (A), lung (B), and liver (C). Elevated MPO activities were observed in the heart, lung, and liver after HS. MPO activity in the Kae PT group was significantly decreased compared with the HS and Kae T groups. Compared with the other groups, ${ }^{\#} P<0.05$, ${ }^{\# \#} P<0.01$. 


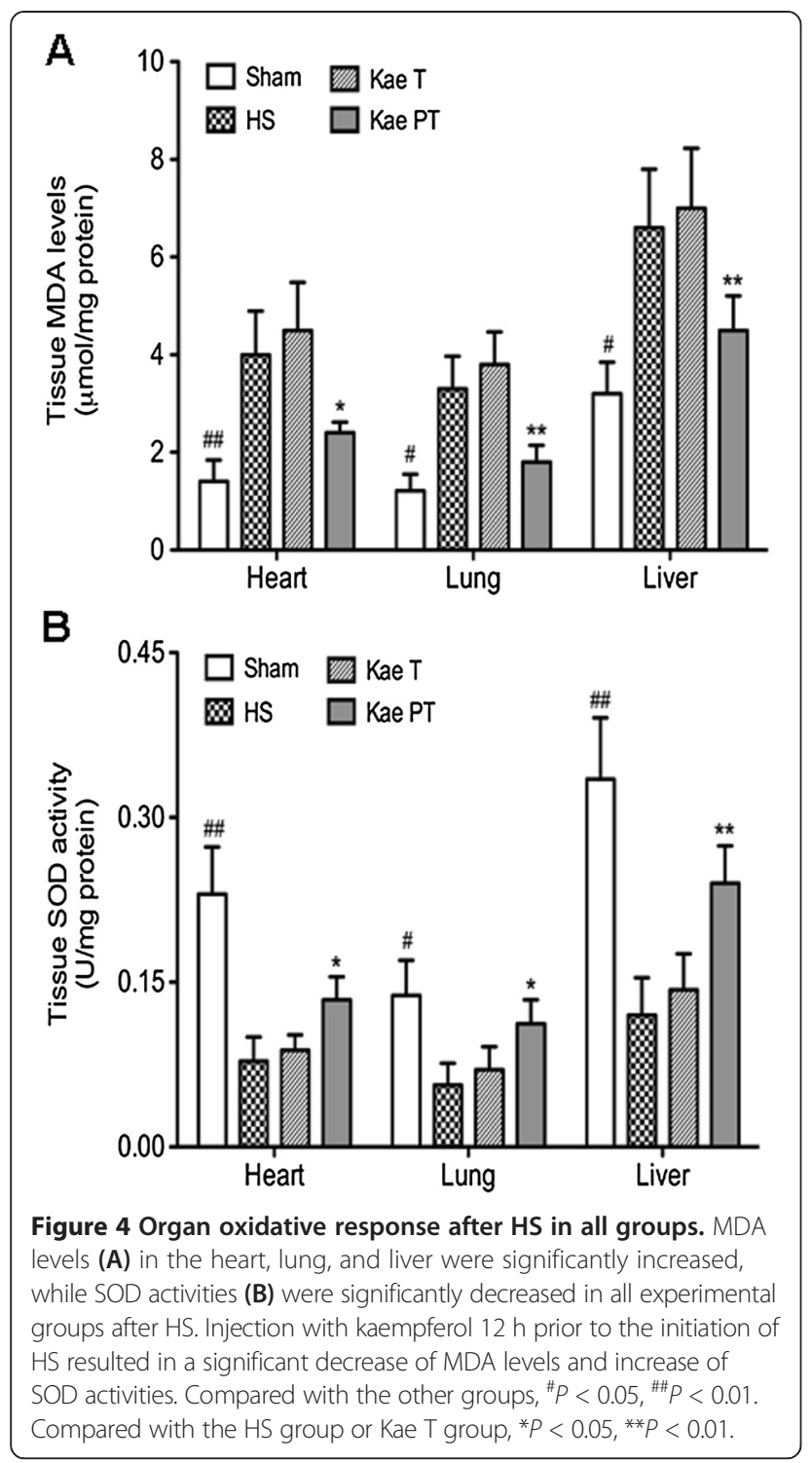

\section{Discussion}

In this study, we investigated the protective effects of kaempferol in HS in mice. The injection of kaempferol prior to the initiation of HS significantly decreased serum proinflammatory cytokines and organ inflammation. Moreover, kaempferol showed its antioxidant ability through inhibiting MDA expression and promoting SOD activities in the heart, lung, and liver.

HS and subsequent ischemia/reperfusion injury are associated with oxidative stress in organs $[18,19]$, and it has been proposed that this generated oxidative stress is a fundamental mechanism of organ damage in HS [20]. Oxygen-free radicals are involved in HS and subsequent resuscitation [21]. Wu et al. [22] confirmed that HS and resuscitation induced significant oxidation in rat lungs, while Panteli et al. [23] reported that the oxidative state of the kidneys remained unaffected after HS. Treatment with antioxidant agents after HS reduced organ oxidative stress and led to a better outcome. Antioxidant therapy with Tempol, a membrane-permeable radical scavenger, improved outcome after HS in rats [24]. In our study, HS and resuscitation induced significant oxidative stress in the heart, lung, and liver, but pretreatment with kaempferol decreased oxidative stress in these organs.

Oxidative stress in HS induces the activation of nuclear factor- $\mathrm{kB}(\mathrm{NF}-\mathrm{kB})$, which triggers an inflammatory cascade during acute hemorrhage, and leads to an overproduction of proinflammatory cytokines [25-27]. Moreover, oxidative stress during HS can induce accumulation and activation of neutrophils and subsequent increases in concentrations of inflammatory mediators, including TNF- $\alpha$ and IL-6, in organs $[28,29]$. The TNF- $\alpha$ and IL-6 plasma levels were significantly increased after HS in our study. In addition, the i.p. injection of kaempferol at a single dose $12 \mathrm{~h}$ prior to the initiation of HS was associated with significantly decreased organ inflammation. This finding was possibly a consequence of the reduced

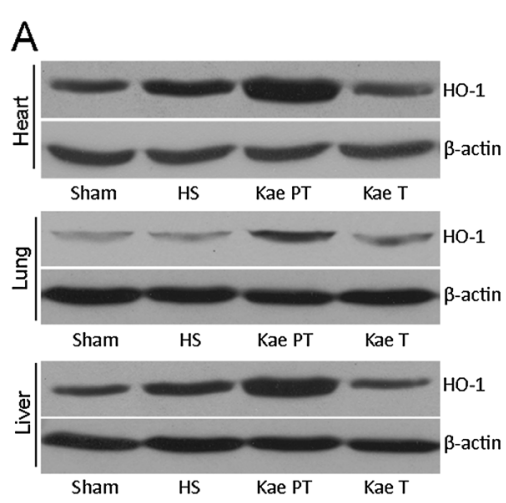

B

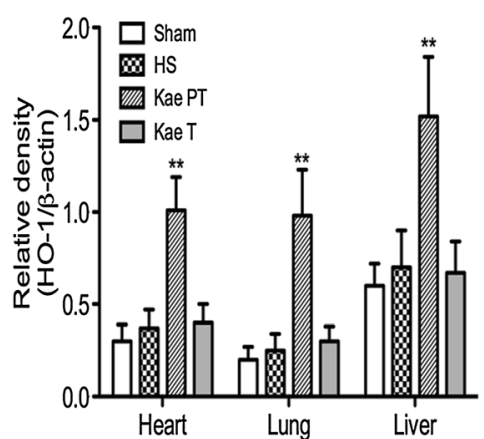

Figure 5 HO-1 expression in heart, lung, and liver after HS. A, Western blot assay for HO-1 expression; $\mathbf{B}$, relative density (HO-1/ $\beta$-actin). Injection with kaempferol $12 \mathrm{~h}$ prior to the initiation of $\mathrm{HS}$ resulted in a significant increase of HO-1 expression in the heart, lung, and liver. Compared with the other groups, ${ }^{* *} P<0.01$. 
systemic inflammatory response observed in our experiment. One previous study found that the activation of NF- $k B$ could be attenuated by treatment with kaempferol [30]. Therefore, its anti-inflammatory and antioxidant abilities in this study were possibly associated with the inhibition of NF-kB expressions after HS.

Modulation of HO-1, also known as heat shock protein 32 , is a promising approach to the attenuation of organ damage [31]. HO-1 is a stress-inducible protein, and its overexpression protects against ischemia-reperfusion injury [32]. Up-regulation of $\mathrm{HO}-1$ could lead to remarkable cytoprotective effects and attenuate organ damage after HS [33]. In addition, kaempferol protected cells against apoptosis via inductions of HO-1 expression [34]. In this study, kaempferol pretreatment resulted in significantly higher levels of HO-1 in major organs including the heart, lung, and liver. Future studies are needed to investigate whether kaempferol also exerts its protective effects via the up-regulation of $\mathrm{HO}-1$ in tissues after HS.

\section{Conclusions}

Pretreatment with kaempferol of HS mice significantly decreased plasma levels of TNF- $\alpha$ and IL-6; reverted MPO, SOD, and MDA in the heart, lung, and liver; and increased expression of $\mathrm{HO}-1$ in the same organs.

\section{Abbreviations}

BW: Body weight; ECL: Enhanced chemiluminescene; ELISA: Enzyme-linked immunosorbent assay; HS: Hemorrhagic shock; HO-1: Heme oxygenase-1; IL-1ß: Interleukin-1 beta; IP: Intraperitoneal; MAP: Mean arterial pressure; MDA: Malondialdehyde; MPO: Myeloperoxidase; NF-kB: Nuclear factor-kappa B; PBS: Phosphate-buffered saline; SOD: Superoxide dismutase; TNF-a: Tumor necrosis factor-alpha.

\section{Competing interests}

The authors declare that they have no competing interests.

\section{Authors' contributions}

YZ and QSY conceived and designed the study. ZXL, LPH, JS, PX, and SZN carried out the study and the data analysis. QSY and XLZ wrote and revised the manuscript. All authors read and approved the final version of the manuscript.

\section{Acknowledgements}

This study was supported by the Emergency Diagnostic \& Therapeutic Center of Central China (Wuhan, Hubei, China).

\section{Author details \\ 'Emergency Center, Zhongnan Hospital of Wuhan University, Wuhan, Hubei 430071, China. ${ }^{2}$ Department of Anesthesiology, Tong Cheng Hospital, Tongcheng, Hubei 437400, China.}

Received: 26 May 2014 Accepted: 6 March 2015

Published online: 21 March 2015

\section{References}

1. Krug EG, Sharma GK, Lozano R. Global burden of injuries. Am J Public Health. 2000;90:523-6.

2. Pfeifer R, Tarkin IS, Rocos B, Pape HC. Patterns of mortality and causes of death in polytrauma patients-has anything changed? Injury. 2009;40:907-11.

3. Peitzman AB, Billiar TR, Harbrecht BG, Kelly E, Udekwu AO, Simmons RL. Hemorrhagic shock. Curr Probl Surg. 1995;32:925-1002.
4. Piccinini AM, Midwood KS. DAMPening Inflammation by Modulating TLR Signalling. Mediators Inflamm. 2010;2010:672395.

5. Arslan F, Keogh B, McGuirk P, Parker AE. TLR2 and TLR4 in ischemia reperfusion injury. Mediators Inflamm. 2010;2010:704202.

6. Chai W, Wang $Y$, Lin JY, Sun XD, Yao LN, Yang YH, et al. Exogenous hydrogen sulfide protects against traumatic hemorrhagic shock via attenuation of oxidative stress. J Surg Res. 2012;176:210-9.

7. Rixen D, Siegel JH. Bench-to-bedside review: Oxygen debt and its metabolic correlates as quantifiers of the severity of hemorrhagic and posttraumatic shock. Crit Care. 2005:9:441-53.

8. Kan WH, Hsieh CH, Schwacha MG, Choudhry MA, Raju R, Bland Kl, et al. Flutamide protects against trauma-hemorrhage-induced liver injury via attenuation of the inflammatory response, oxidative stress, and apoptosis. J Appl Physiol. 2008;105:595-602.

9. Hildebrand F, Hubbard WJ, Choudhry MA, Frink M, Pape HC, Kunkel SL, et al. Kupffer cells and their mediators: The culprits in producing distant organ damage after trauma-hemorrhage. Am J Pathol. 2006;169:784-94.

10. Park MY, Ji GE, Sung MK. Dietary kaempferol suppresses inflammation of dextran sulfate sodium-induced colitis in mice. Dig Dis Sci. 2012;57:355-63.

11. Kowalski J, Samojedny A, Paul M, Pietsz G, Wilczok T. Effect of apigenin, kaempferol and resveratrol on the expression of interleukin-1b and tumor necrosis factor-a genes in J774.2 macrophages. Pharmacol Rep. 2005;57:390-4.

12. Chen AY, Chen YC. A review of the dietary flavonoid, kaempferol on human health and cancer chemoprevention. Food Chem. 2013;138:2099-107.

13. Cortes JR, Perez-G M, Rivas MD, Zamorano J. Kaempferol inhibits IL-4-induced STAT6 activation by specifically targeting JAK3. J Immunol. 2007;179:3881-7.

14. De Groot H, Rauen U. Tissue injury by reactive oxygen species and the protective effects of flavonoids. Fundam Clin Pharmacol. 1998;12:249-55.

15. Sergediene E, Jönsson K, Szymusiak H, Tyrakowska B, Rietjens IM. Cenas. Pro-oxidant toxicity of polyphenolic antioxidants to HL-60 cells description of quantitative structure activity relationships. FEBS Lett. 1999;462:392-6.

16. Kobbe $P$, Lichte $P$, Schreiber $H$, Reiss LK, Uhlig S, Pape HC, et al. Inhalative IL-10 attenuates pulmonary inflammation following hemorrhagic shock without major alterations of the systemic inflammatory response. Mediators Inflamm. 2012;2012:512974.

17. Pfeifer $R$, Lichte $P$, Schreiber $H$, Sellei RM, Schmidt J, Dombroski $D$, et al. Inhalative vs. systemic IL-10 administration: Differences in the systemic inflammatory response and end-organ inflammation following hemorrhagic shock. Cytokine+. 2012;60:266-70.

18. Jaeschke H, Farhood A. Neutrophil and Kupffer cell-induced oxidant stress and ischemia-reperfusion injury in rat liver. Am J Physiol. 1991;260:G355-62.

19. Menezes J, Hierholzer C, Watkins SC, Lyons V, Peitzman AB, Billiar TR, et al. A novel nitric oxide scavenger decreases liver injury and improves survival after hemorrhagic shock. Am J Physiol. 1999;277:G144-51.

20. Jarrar D, Wang P, Cioffi WG, Bland Kl, Chaudry IH. Critical role of oxygen radicals in the initiation of hepatic depression after trauma hemorrhage. J Trauma. 2000:49:879-85.

21. Broechner AC, Toft P. Pathophysiology of the systemic inflammatory response after major accidental trauma. Scand J Trauma Resusc Emerg Med. 2009;17:43

22. Wu BG, Peng TC, Tsai PS, Wang TY, Jeng MJ, Huang CJ. High-lipid enteral nutrition could partially mitigate inflammation but not lung injury in hemorrhagic shock rats. J Surg Res. 2013;184:997-1005.

23. Panteli ES, Fligou F, Papamichail C, Papapostolou I, Zervoudakis G, Georgiou $C D$, et al. Quantification of superoxide radical production in 4 vital organs of rats subjected to hemorrhagic shock. Am J Emerg Med. 2012;30:476-80.

24. Kentner R, Safar P, Behringer W, Wu X, Kagan VE, Tyurina YY, et al. Early Antioxidant Therapy with Tempol during Hemorrhagic Shock Increases Survival in Rats. J Trauma. 2002;53:968-77.

25. Altavilla D, Saitta A, Guarini S, Galeano M, Squadrito G, Cucinotta D, et al. Oxidative stress causes nuclear factor-kappaB activation in acute hypovolemic hemorrhagic shock. Free Radic Biol Med. 2001;30:1055-66.

26. Blackwell TS, Christman JW. The role of nuclear factor-kappa B in cytokine gene regulation. Am J Respir Cell Mol Biol. 1997;17:3-9.

27. Guarini S, Altavilla D, Cainazzo MM, Giuliani D, Bigiani A, Marini $H$, et al Efferent vagal fibre stimulation blunts NF-kB activation and protects against hypovolemic hemorrhagic shock. Circulation. 2003;107:1189-94.

28. Tasoulis MK, Livaditi $O$, Stamatakos M, Stefanaki $C$, Paneris $P$, Prigouris $P$, et al. High concentrations of reactive oxygen species in the BAL fluid are correlated with lung injury in rabbits after hemorrhagic shock and resuscitation. Tohoku J Exp Med. 2009;219:193-9. 
29. Shenkar R, Abraham E. Mechanisms of lung neutrophil activation after hemorrhage or endotoxemia: roles of reactive oxygen intermediates, NF-kappa B, and cyclic AMP response element binding protein. J Immunol. 1999;163:954-62.

30. Crespo I, García-Mediavilla MV, Gutiérrez B, Sánchez-Campos S, Tuñón MJ, González-Gallego J. A comparison of the effects of kaempferol and quercetin on cytokine-induced pro-inflammatory status of cultured human endothelial cells. Br J Nutr. 2008;100:968-76.

31. Nakao A, Kaczorowski DJ, Sugimoto R, Billiar TR, McCurry KR. Application of Heme Oxygenase-1, Carbon Monoxide and Biliverdin for the Prevention of Intestinal Ischemia/Reperfusion Injury. J Clin Biochem Nutr. 2008;42:78-88.

32. Gueler F, Park JK, Rong S, Kirsch T, Lindschau C, Zheng W, et al. Statins attenuate ischemia-reperfusion injury by inducing heme oxygenase- 1 in infiltrating macrophages. Am J Pathol. 2007;170:1192-9.

33. Lu YQ, Gu LH, Jiang JK, Mou HZ. Effect of hypertonic versus isotonic saline resuscitation on heme oxygenase-1 expression in visceral organs following hemorrhagic shock in rats. Biomed Environ Sci. 2013;26:684-8.

34. Gao SS, Choi BM, Chen XY, Zhu RZ, Kim Y, So H, et al. Kaempferol suppresses cisplatin-induced apoptosis via inductions of heme oxygenase- 1 and glutamate-cysteine ligase catalytic subunit in HEl-OC1 cell. Pharm Res. 2010:27:235-45.

\section{Submit your next manuscript to BioMed Central and take full advantage of:}

- Convenient online submission

- Thorough peer review

- No space constraints or color figure charges

- Immediate publication on acceptance

- Inclusion in PubMed, CAS, Scopus and Google Scholar

- Research which is freely available for redistribution 